\title{
Acceptor-induced threshold energy for the optical charging of InAs single quantum dots
}

\author{
E. S. Moskalenko, ${ }^{1,2}$ K. F. Karlsson, ${ }^{1}$ P. O. Holtz, ${ }^{1}$ B. Monemar, ${ }^{1}$ W. V. Schoenfeld ${ }^{3}$ J. M. Garcia, ${ }^{3}$ and P. M. Petroff ${ }^{3}$ \\ ${ }^{1}$ Department of Physics and Measurement Technology, Linköping University, S-581 83 Linköping, Sweden \\ ${ }^{2}$ A. F. Ioffe Physical-Technical Institute, RAS, 194021, Polytechnicheskaya 26, St. Petersburg, Russia \\ ${ }^{3}$ Materials Department, University of California, Santa Barbara, California 93106
}

(Received 10 June 2002; published 27 November 2002)

\begin{abstract}
We study the photoluminescence of single InAs/GaAs self-assembled quantum dots for a range of excitation powers, excitation energies and sample temperatures $4 \mathrm{~K}<T<50 \mathrm{~K}$. Our results demonstrate the existence of a well-defined excitation energy threshold, above which negatively charged excitons could be effectively created in a single quantum dot at helium temperatures. This threshold energy is in the range between the wetting layer ground state energy and the GaAs barrier energy, and is ascribed to the acceptor to the conduction band transition in GaAs. A model is presented, according to which a laser excitation of this energy creates an extra number of free electrons in the GaAs barrier in addition to the equal number of electrons and holes in the wetting layer. The excitation power dependence of these extra electrons has been calculated and found to be in good agreement with the experimentally derived values of the total electron charge, accumulated in the quantum dot. At elevated temperatures $(T>30 \mathrm{~K})$, this effect vanishes due to the essential decrease of the steady-state free electron concentration in the GaAs barrier as a result of thermally excited free holes appearing in the GaAs barrier valence band which provides an effective recombination channel for the free electrons. These experimental observations could be used as an effective tool to create and study charged excitons in quantum dots.
\end{abstract}

DOI: $10.1103 /$ PhysRevB.66.195332

PACS number(s): 73.21.La, 71.55.Eq, 73.22.Dj, 71.70.Gm

\section{INTRODUCTION}

Semiconductor quantum dots (QD's) are of great contemporary interest mainly for their similarities to real atoms in the sense of an effective confinement of electrons $(e)$ and holes $(h)$ on the nanometer length scale in all three directions and hence may be referred to as "artificial atoms" 1 with discrete energy levels for $e$ and $h$. This results in a zerodimensional density of states as well as in an increased importance of Coulomb interactions in QD's. ${ }^{1}$ The latter fact together with the restricted number (typically two) of particles, which could be accommodated in the ground state of the QD, result in considerable multiparticle effects, which are of great importance both from a fundamental physics point of view as well as for a variety of applications. Indeed, multiparticle states, consisting of a large number of $e$ and $h$ (equal or nonequal) in QD's, which have been studied theoretically $^{2-9}$ and experimentally, ${ }^{1,10-21}$ determine the performance of a number of QD's based optoelectronic (electronic) devices, such as QD lasers, ${ }^{22}$ QD infrared detectors, ${ }^{23}$ QD memory devices, ${ }^{24,25}$ and single-electron transistors, which in turn are considerably affected by the charge, stored in the QD ${ }^{25,26}$

This highlights the important role of multiparticle complexes with nonequal number of $e$ and $h$, and hence stimulates the study of charged exciton complexes, which in the simplest case consist of one exciton and one additional charge carrier (i.e., $e$ or $h$ ). The formation of more complex charged excitons in QD's with up to 20 electrons has been considered theoretically. ${ }^{3}$ Also the influence of the electron occupation of the QD on its absorption spectrum ${ }^{7}$ and the effect of dielectric mismatch between the QD and the surrounding material on the QD's quasiparticle energies ${ }^{8,9}$ have been calculated recently. Experimental evidence for the formation of few electron states in QD's was revealed in capacitance experiments ${ }^{14,18}$ and combined studies of capacitance and interband transmission. ${ }^{16,17}$ The formation of negatively charged exciton complexes was demonstrated in photoluminescence (PL) experiments. ${ }^{5,15,17,19,20}$ However, these optical studies have been based on measurements of large ensembles of dots, so the results inevitably include the effect of inhomogeneous broadening, which prevents accurate measurements and hence a detailed analysis of the optical properties of the QD. There is accordingly a strong demand for luminescence studies of charged exciton phenomena in single dots.

In recent years, intensive studies on luminescence of individual QD's have resulted in a number of publications. ${ }^{27-38}$ Several different approaches have been employed to load a single QD with extra charges in these experiments. For instance, a sophisticated sample design has been employed, ${ }^{35}$ in which a layer of low density $\operatorname{In}(\mathrm{Ga})$ As QD's is embedded in a wide GaAs quantum well (QW), while a neighboring more narrow QW separated from the front QW by a rather thin AlAs barrier, serves as an effective supply of extra carriers under optical excitation. Another method is to use samples containing an $n$-doped layer with contacts $^{30,33}$ or samples prepared as a Schotty diodes. ${ }^{34}$ The number of extra electrons in the QD can then be tuned by an applied external voltage. This gives a possibility to control the average number of excess electrons in the dot. However, the applied voltage causes a shift of the entire spectrum and deforms the wave functions, which eventually leads to a change in the interaction energies. This circumstance was mentioned ${ }^{19}$ as a possible reason responsible for the deviation between the 
calculated interaction energies and the experimentally derived energies.

A third way to study charged excitons can be achieved by employing samples, where the QD's are initially filled with a random number of electrons from the background doping. ${ }^{27-29}$ The results obtained have allowed the identification of distinct transitions related to excitons charged with up to six additional electrons. ${ }^{28}$ However, the drawback of this method is that the initial number of excess electrons depends on the arbitrary number of impurity atoms in the close vicinity of the investigated QD and hence hardly controllable.

In our previous publications, ${ }^{31,32}$ we have suggested a new method to create and study negatively charged excitons in InAs/GaAs self-assembled QD's by pure optical means. This was based on the idea that the QD effectively collects the free carries, photocreated with an excitation energy $h \nu_{\mathrm{ex}}$ higher than the band gap energy $E_{g}^{\mathrm{GaAs}}$ of the GaAs barrier. Hence the carrier diffusivity considerably depends on its kinetic energy, ${ }^{39}$ one can expect that as $h \nu_{\mathrm{ex}}$ is tuned, the markedly different diffusivities of excited $e$ 's and $h$ 's would result in a faster capture of $e$ 's into the QD. This effect resulted in the appearance of two new lines in micro PL ( $\mu$-PL) spectra, redshifted relative to the neutral exciton and interpreted as the negatively charged excitons, while only the neutral exciton was detected, when the excitation energy was resonant with the wetting layer (WL) ground state $E_{\mathrm{WL}} \cdot{ }^{31}$ The observations of a PL line, blueshifted with respect to the neutral exciton and interpreted as positively charged exciton, were reported also for InAs/GaAs QD's in the case of $h \nu_{\mathrm{ex}}$ $>E_{\mathrm{WL}}$ (Ref. 37) and $h \nu_{\mathrm{ex}}>E_{g}^{\mathrm{GaAs}},{ }^{36,38}$ respectively.

In the present contribution, we report on a sharp threshold for $h \nu_{\mathrm{ex}}$ in the range of $E_{\mathrm{WL}}<h \nu_{\mathrm{ex}}<E_{g}^{\mathrm{GaAs}}$ in the $\mu$-PL spectra for the formation of the negatively charged exciton, in contrast to the results of Ref. 37, where the charged exciton line was detected at any $h \nu_{\mathrm{ex}}>E_{\mathrm{WL}}$. To explain the existence of this threshold energy $h \nu_{\text {th }}$ we propose a model, according to which the laser excitation in the range of $E_{\mathrm{WL}}$ $<h \nu_{\mathrm{ex}}<E_{g}^{\mathrm{GaAs}}$, creates an equal number of $e$ 's and $h$ 's in the WL region. In addition, extra electrons originating from the acceptor atoms, positioned in the GaAs barrier at some distances from the QD (which are restricted, of course, by the radius of the laser spot $\leqslant 1 \mu \mathrm{m}$ ) are excited. Since electrons, once being photoexcited in GaAs, are able to move over a distance of several $\mu$ m's at helium temperatures, ${ }^{39}$ they are effectively captured into the QD, initiating the appearance of negatively charged exciton lines.

We would like to stress here that our results are in sharp contrast to the findings of Ref. 28, where the QD under study was found to be negatively charged without laser illumination (as a result of the electrons capture into the QD from the donor atoms, positioned in the close vicinity of the QD) and became more neutral as a result of "photodepletion" processes, when the laser was switched on. In our case, on the other hand, the appearance of negative charge in the QD is demonstrated as a result of laser excitation. In addition, a necessary condition for the QD charging from impurity/ defect atoms in Refs. 28, 29 was the rather short distance [typically not longer than $10 \mathrm{~nm}$ (Ref. 40)] between the QD and the defect atom, providing the nonvanishing probability for the electron to tunnel from the defect atom into the QD. Performing the spatially resolved measurements, by shifting away the laser spot from a QD, we have succeeded to prove experimentally that in our case, the QD's under study are able to collect carriers photocreated at rather large distances $(\sim 2.5 \mu \mathrm{m})$ from the QD's, thus avoiding the abovementioned necessary condition for the QD's charging.

We also present calculations of the steady-state concentration of extra electrons photoexcited from the acceptor atoms as a function of the excitation power $P_{\text {ex }}$ and crystal temperature $T$. The theoretical predictions are found to be in good agreement with the experimentally derived amount of extra charge accumulated in the QD. We suggest that the presently proposed method (complementary to the previously presented ${ }^{31}$ ) to create charged excitons in the QD could be widely used in practice as an effective tool to study "artificial atoms" populated with a nonequal amount of electrons and holes.

\section{SAMPLES AND EXPERIMENTAL SETUP}

The samples studied were grown by molecular beam epitaxy on a semiinsulating GaAs (100) substrate. The buffer layer was prepared with a short-period superlattice 40 $\times 2 \mathrm{~nm} / 2 \mathrm{~nm} \mathrm{AlAs} / \mathrm{GaAs}$ at a growth temperature of $630^{\circ} \mathrm{C}$. On top of a 100-nm GaAs layer the QD's were formed from a 1.7 InAs monolayers layer deposited at $530{ }^{\circ} \mathrm{C}$. A first growth interruption of $30 \mathrm{sec}$ was used to improve the size distribution. Then the dots were covered with a thin GaAs cap layer with a thickness of $t_{\text {cap }}=3 \mathrm{~nm}$ before a crucial second growth interruption of $30 \mathrm{sec}$. Finally, a 100-nm-thick GaAs layer was deposited to protect the QD's. Transmission electron microscopy studies of analogously grown samples revealed that uncapped original dots are lens (hemispherical) shaped with a typical lateral size of $35 \mathrm{~nm}$ and a height of 10 $\mathrm{nm} .{ }^{41}$ The deposition of a GaAs capping layer after that the dots have been formed with $t_{\text {cap }}=3 \mathrm{~nm}$ leads to an essential reduction in the QD's height down to $4.5 \mathrm{~nm}$. Consequently, the PL is blueshifted to the spectral region of $1.34 \mathrm{eV}(\sim 950$ $\mathrm{nm},{ }^{41}$ i.e., within the sensitivity spectral range of the SiCCD camera. The sample was grown without rotation of the substrate, so that a gradual variation of In flux is achieved across the wafer resulting in a gradient in both the density and the average size of the dots across the epitaxial layer. ${ }^{41}$

The QD's were studied by means of a conventional diffraction-limited $\mu$-PL setup. To excite the QD's, we used a $\mathrm{cw}-\mathrm{Ar}$ laser pumped Ti-Sp laser, tunable between 700 to $1000 \mathrm{~nm}$, which power was adjusted by the use of a continuously graded neutral density filter. The beam of the Ti-Sp laser was focused on the sample surface by a microscope objective through a thin optical window of the continuous flow cryostat. The lowest temperature available was $3.8 \mathrm{~K}$ and could be increased up to $100 \mathrm{~K}$. The laser beam could be focused on the sample surface down to a spot size of $2 \mu \mathrm{m}$ in diameter. The luminescence signal was collected by the same objective and dispersed by a single-grating $0.45-\mathrm{m}$ monochromator combined with a $\mathrm{LN}_{2}$ cooled $\mathrm{Si}-\mathrm{CCD}$ camera. The 
spectral resolution achieved in the region of the studied PL was $0.15 \mathrm{meV}$. For the PL excitation (PLE) measurements, a double-grating $0.85-\mathrm{m}$ monochromator was used in combination with a $\mathrm{LN}_{2}$ cooled InGaAsP photomultiplier.

To find the particular QD to study, a laser beam was scanned across the sample surface. Once the desired QD was found, special marks (grids) were fabricated on the sample surface around the QD with the laser beam of very high power density. This allowed us to estimate the average distance between the adjacent QD's to be around $10 \mu \mathrm{m}$ in the studied QD structure. To control the exact position of the laser spot on the sample surface, the image of the interesting sample region was projected by a video camera, which made it easy to find the desired QD by using the fabricated marks. In addition, this arrangement allowed us to effectively correct the laser position on the sample, if the sample was moved due to the thermal drift. It should be noted that with this method to locate the exact QD position by using the described grids, one can avoid some undesirable consequences, which take place with other methods. For example, when a metal mask with small holes is deposited on top of the sample, this metal mask may produce an electric field in the near-surface region of the sample and act as a stressor, which could spoil the entire quality of the QD's.

Fourteen single QD's located at different spatial positions of the sample were examined in this study. All of them revealed an analogous behavior [what concerns the PL spectra evolution with $P_{\mathrm{ex}}$ and $T$ at $\left.h \nu_{\mathrm{ex}}>(<) h \nu_{\mathrm{th}}\right]$, except for the exact value of $h \nu_{\text {th }}$, which could be achieved at highest $P_{\mathrm{ex}}$. With respect to this difference, all QD's studied can be divided into two groups: One group, which at highest $P_{\mathrm{ex}}$ shows $h \nu_{\text {th }}$ at an energy of $1.483 \mathrm{eV}$ and another group with $h \nu_{\text {th }}$ at $1.493 \mathrm{eV}$. For consistency, we present the PL spectra taken for only one QD1 (chosen from the first group) and compare the data of $h \nu_{\text {th }}$ as a function of $P_{\text {ex }}$ with those taken for the other QD2 (chosen from the second group).

\section{EXPERIMENTAL RESULTS AND DISCUSSION}

Figure 1 a shows PL spectra of an individual dot QD1, taken at a temperature of $T=4.1 \mathrm{~K}$ and an excitation power, $P_{\mathrm{ex}}=50 \mathrm{nW}$, for a number of excitation energies $E_{\mathrm{WL}}$ $<h \nu_{\mathrm{ex}}<E_{g}^{\mathrm{GaAs}}$, where $E_{\mathrm{WL}}$ and $E_{g}^{\mathrm{GaAs}}$ correspond to the maximum of the wetting layer PL emission [shown as a solid line in Fig. 1(b)] and the band gap energy of the GaAs barrier $(1.519 \mathrm{eV})$, respectively. The excitation energies used for each of the PL spectra, correspond to the zero-level position of the particular spectrum relative to the vertical axis of the panel (b) in Fig. 1. Altogether three emission lines, marked as $X, X^{-}$, and $X^{--}$in Fig. 1(a), peaking at $1.3406 \mathrm{eV}$, $1.3375 \mathrm{eV}$, and $1.3328 \mathrm{eV}$, respectively, could be detected with an intensity distribution depending on the exact value of the $h \nu_{\mathrm{ex}}$. The detailed analysis of the origins of these spectral lines, made by us previously, ${ }^{31}$ allows one to ascribe these to the neutral $(X)$, the single negatively charged $\left(X^{-}\right)$, and the doubly negatively charged $\left(X^{--}\right)$exciton complexes, consisting of the $1 e 1 h, 2 e 1 h$, and $3 e 1 h$ configurations, respectively.

Evidently, the PL spectra undergo a considerable evolu-
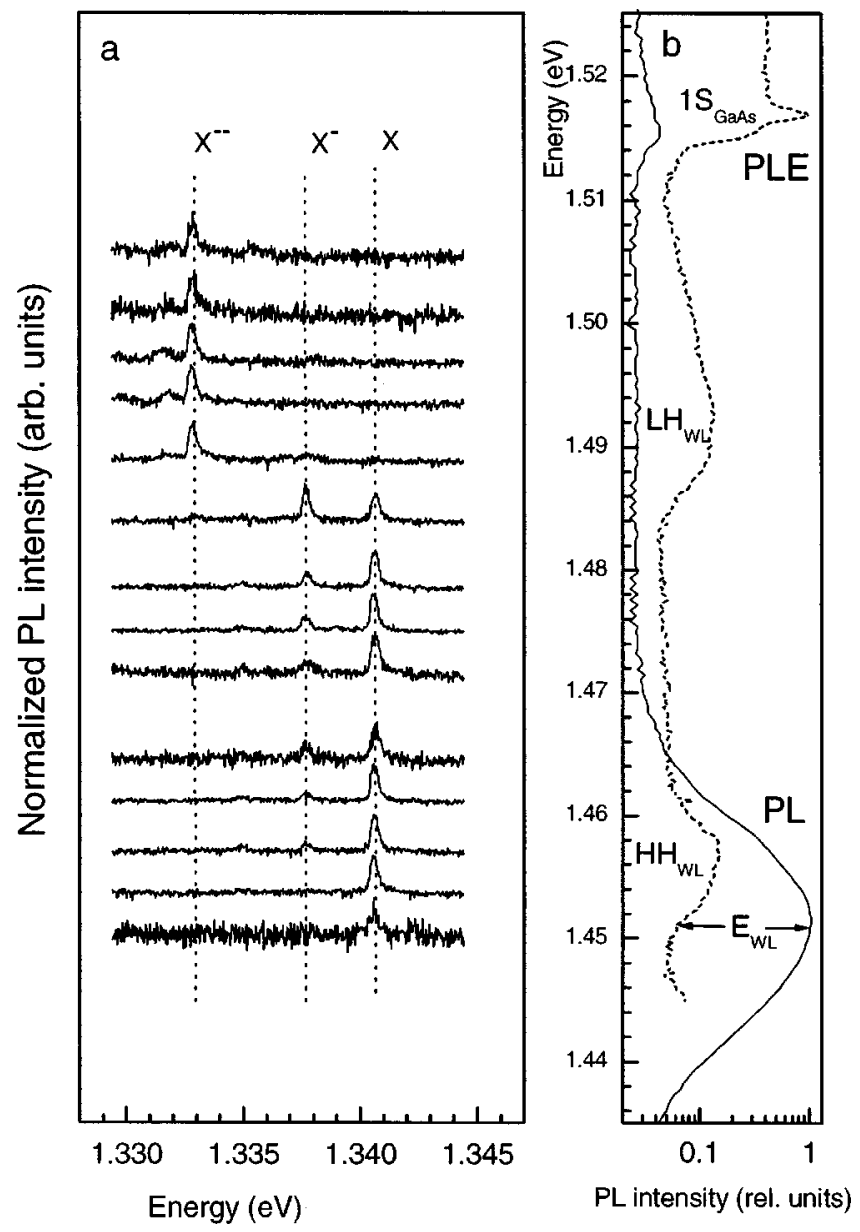

FIG. 1. (a) PL spectra of a single QD1 taken at $T=4.1 \mathrm{~K}$, for an excitation power $P_{\mathrm{ex}}=50 \mathrm{nW}$ and a number of excitation energies, which correspond to the zero-level position of the particular spectrum relative to the vertical axis of the panel (b). All spectra are normalized at the maximum value of their PL amplitude and are vertically shifted for clarity. (b) Normalized macro-PL (solid line) and macro-PLE (dotted line) spectra taken from a large $(50 \mu \mathrm{m})^{2}$ sample area around the QD1 position at $T=4.1 \mathrm{~K}$ and $P_{\text {ex }}$ $=50 \mathrm{~mW}$. The PLE spectrum is obtained by detecting at $1.441 \mathrm{eV}$ and $h \nu_{\mathrm{ex}}=1.675 \mathrm{eV}$ was used to excite the PL spectrum.

tion with the increase of $h \nu_{\mathrm{ex}}$ in the energy range between 1.484 and $1.490 \mathrm{eV}$, with a progressive intensity redistribution in favor of the $X^{-}$and $X^{--}$lines at the expense of the $X$ line. All PL spectra with $1.490 \mathrm{eV}<h \nu_{\mathrm{ex}}<1.510 \mathrm{eV}$ entirely consist of the $X^{--}$line, indicating the QD has been loaded with two extra electrons. Thus, the spectra in Fig. 1 reveal the existence of a distinct threshold energy $h \nu_{\text {th }}$, above (below) which the QD is in the negatively charged (neutral) state, respectively. In what follows, we will analyze the origin of this threshold energy and its dependence on the experimental conditions, such as $P_{\mathrm{ex}}$ and $T$.

First of all, we would like to note that the $h \nu_{\mathrm{ex}}$ value, which could be derived from the data shown in Fig. 1(a), coincides in energy with the peak denoted as $\mathrm{LH}_{\mathrm{WL}}$ in the PLE spectrum of the wetting layer shown in Fig. 1(b) as a dashed line. The origin of this peak is claimed to be due to 


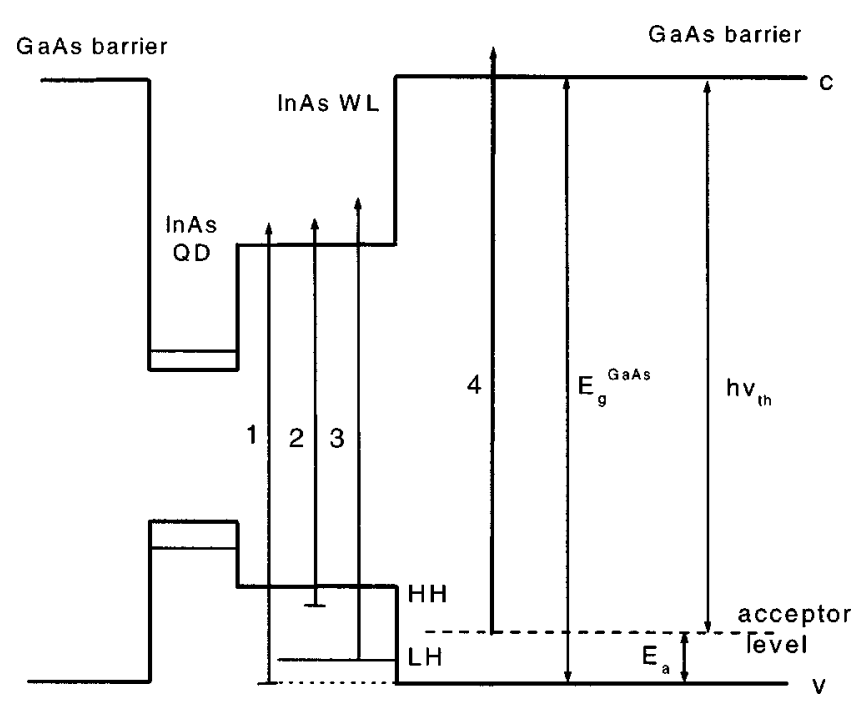

FIG. 2. Conduction $(c)$ and valence $(v)$ band profiles and the acceptor level position in the studied sample. Arrows 1, 2, 3, and 4 indicate the free-to-bound, heavy-hole, light-hole, and acceptor-toconduction band transitions, respectively. Also the energies of the GaAs band gap $\left(E_{g}^{\mathrm{GaAs}}\right)$, the threshold energy $\left(h \nu_{\mathrm{th}}\right)$, and the acceptor binding energy $\left(E_{a}\right)$ are shown.

the transition from the valence band of the GaAs barrier to the electron level in the WL (arrow 1 in Fig. 2). With this interpretation, the appearance of extra electrons in the WL could be understood as follows. At $h \nu_{\text {ex }}>h \nu_{\text {th }}$, an equal number of $e$ 's and $h$ 's is excited in the WL due to transitions between the heavy hole $(\mathrm{HH})$ and the conduction band (shown as arrow 2 in Fig. 2). In addition, free holes can be excited (together with electrons from the WL's conduction band) in the valence band of the GaAs barrier (arrow 1 in Fig. 2) in the close vicinity of the WL. These holes will move away from the WL region, forced by the internal electric field (which could be as much as $10^{4} \mathrm{~V} / \mathrm{cm}$, as shown in analogously grown samples), ${ }^{15}$ thus effectively populating the WL (and hence the QD) with an extra amount of electrons.

To confirm or deny this explanation, we compare the $h \nu_{\text {th }}$ values, obtained for different QD's with the details of the corresponding PLE spectra, registered from a very small sample area $(2 \mu \mathrm{m})^{2}$ around each QD. This small area contains only one QD, in contrast to the case illustrated in Fig. 1(b), where the PLE spectrum was detected from a larger (50 $\mu \mathrm{m})^{2}$ part of the sample. The threshold energies for the QD1 (QD2) are given in Fig. 3(a) by solid (open) symbols for a range of $P_{\text {ex }}$ 's. The dependence of $h \nu_{\text {th }}$ on $P_{\text {ex }}$ will be discussed below. Figure 3(b) shows an example of the experimentally derived $h \nu_{\mathrm{ex}}$ value for QD1 (marked by an arrow) from the plot of the amplitudes of the $X$ and $X^{--}$PL lines on its dependence on the $h \nu_{\mathrm{ex}}$, taken for $T=4.1 \mathrm{~K}$ and $P_{\mathrm{ex}}$ $=50 \mathrm{nW}$ [as in Fig. 1(a)]. The comparison of the $h \nu_{\text {th }}$ values obtained for the QD1 and QD2 together with the details of the corresponding $\mu$-PLE spectra shown in Fig. 3(c), reveals that there is not any one-to-one correspondence between the $h \nu_{\text {th }}$ values and the spectral features of the peaks, marked as $\mathrm{LH}_{\mathrm{WL}}$. Hence, the immediate conclusion to be drawn from these findings, is that the threshold-related phenomenon and
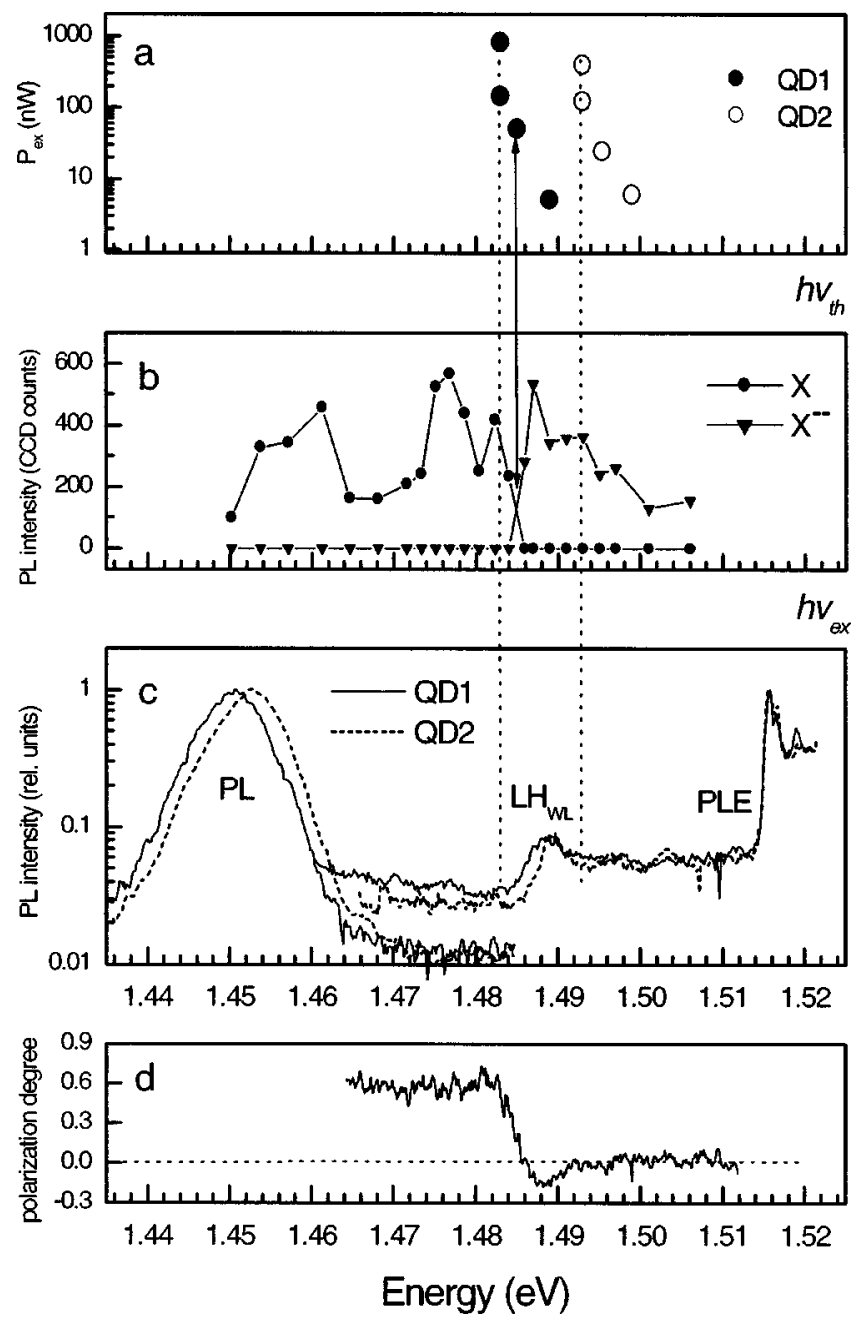

FIG. 3. (a) The threshold energy $h \nu_{\text {th }}$ positions taken at $T$ $=4.1 \mathrm{~K}$ and for a number of $P_{\mathrm{ex}}$ for two different QD's: QD1 (solid symbols) and QD2 (open symbols). (b) The amplitudes of the $X$ and $X^{--}$PL lines as a function of the excitation energy $h \nu_{\mathrm{ex}}$ taken at $T=4.1 \mathrm{~K}$ and $P_{\mathrm{ex}}=50 \mathrm{nW}$ for QD1. The arrow shows the $h \nu_{\text {th }}$ value derived from this plot. (c) The normalized $\mu$-PL and $\mu$-PLE spectra taken from a small $(2 \mu \mathrm{m})^{2}$ sample area around QD1 (solid lines) and QD2 (dotted lines) at $T=4.1 \mathrm{~K}$ and $P_{\text {ex }}=0.1 \mathrm{~mW}$. PLE spectra were obtained by detecting at the maximum of the corresponding PL lines. The PL spectra were excited at $h \nu_{\mathrm{ex}}$ $=1.530 \mathrm{eV}$. (d) The degree of polarization derived from the cleaved-edge $\mu$-PLE spectra (see text for the details) registered at the same conditions as in (c). The left (right) vertical dotted line shows the $h \nu_{\text {th }}$ value achieved at highest $P_{\text {ex }}$ for the QD1 (QD2), respectively.

the peak, denoted as $\mathrm{LH}_{\mathrm{WL}}$, have completely different origins.

To further elucidate the possible role of the $\mathrm{LH}_{\mathrm{WL}}$ peak on the results obtained, we performed polarized cleavedsample-edge measurements, which allowed us to identify the character (heavy or light hole) of this peak. We first note that although the spatial separation between QD1 and QD2 is several $\mathrm{mm}$, the $\mu$-PL and $\mu$-PLE spectra in Fig. 3(c) are almost identical, except for the small shift $(\approx 2 \mathrm{meV})$ for both spectra, which is explained by slightly different average 
thicknesses of the WL in the vicinity of these QD's. Secondly, the $\mu$-PLE and the macro-PLE spectra of QD1 shown in Fig. 3(c) (solid line) and Fig. 1(b) (dotted line), respectively, are similar. These two circumstances imply that the features, obtained in the cleaved-edge experiments, performed at the sample edge, are identical to those revealed in $\mu$-PLE spectra of Fig. 3(c), which were detected from the sample surface.

The cleaved-edge $\mu$-PLE experiments were performed in a geometry such that the laser light was focused on the sample edge, which was cut prior to the measurements. $\mu$-PLE spectra were measured at two linear polarizations $\left(e_{\|}\right.$ and $e_{\perp}$ ) of the exciting light, parallel and perpendicular to the plane of the WL, respectively. We exploit here the absorption selection rules valid for the InAs quantum wells, ${ }^{42}$ namely, that the absorption due to transitions involving lightholes (LH's) should be stronger for $e_{\perp}$ than for $e_{\|}$while the opposite situation should apply for the heavy holes (HH's). Consequently, the $\mathrm{HH}(\mathrm{LH})$ character can readily be obtained from a positive (negative) value of the polarization degree $\left(I_{\|}-I_{\perp}\right) /\left(I_{\|}+I_{\perp}\right)$, where $I_{\|}$and $I_{\perp}$ are the PLE intensities registered in the $e_{\|}$and $e_{\perp}$ cases, respectively. The polarization degree, measured by PLE at the sample edge position, equally spaced with a similar spatial separation from QD1 and QD2, is shown in Fig. 3(d). As seen from this figure, the $\mathrm{LH}_{\mathrm{WL}}$ peak exhibits an obvious LH character (arrow 3 in Fig. 2), while the transitions at lower energies have $\mathrm{HH}$ character (arrow 2 in Fig. 2). Consequently, when $h \nu_{\mathrm{ex}}$ is tuned into resonance with the $\mathrm{LH}_{\mathrm{WL}}$ peak, an equal number of $e$ 's and $h$ 's are created in the WL and, as a result, no extra electrons can appear. As a result, any association between this peak and the $h \nu_{\text {th }}$ can be ruled out.

Another possible explanation of the $h \nu_{\text {th }}$ origin is based on the dependence of the carrier relaxation and transport processes on the carrier interaction with the optical phonons (LO). In fact, in our previous work, ${ }^{31}$ we exploited this circumstance and found a well-defined periodic (with the period of $41.4 \mathrm{meV}$ ) counterphase evolution of the PL amplitudes for the $X$ and $X^{--}$lines as a function of $h \nu_{\mathrm{ex}}$ $\left(>E_{g}^{\mathrm{GaAs}}\right)$. The present experimental findings, on the contrary, do not exhibit any periodic evolution (in $h \nu_{\mathrm{ex}}$ ) of the PL spectra, but reveal a very narrow (few meV's) energy range of $h \nu_{\text {ex }}$ 's, in which the PL spectra undergo a complete transformation. Accordingly, some other mechanism has to be taken into account to explain the charging of the excitons in the present experiments.

LO-assisted relaxation processes could also be considered as important, when the energy difference between $h \nu_{\mathrm{ex}}$ and the WL ground state or the QD ground (excited) state exactly matches an integer number of LO phonon energies, as was experimentally demonstrated in Ref. 43 for the case of InAs/ GaAs QDs. The experimental results show that this mechanism can not be used to explain the $h \nu_{\text {th }}$ existence in our case. This fact is based on the following findings. (i) $h \nu_{\text {th }}$ energies acquire different values, by $10 \mathrm{meV}$, for the two groups of QD's [Fig. 3(a)], while their $\mu$-PLE spectra differ significantly less, by $2 \mathrm{meV}$ [Fig. 3(c)]. Hence, the resonant excitation with respect to the WL ground state can be ex-
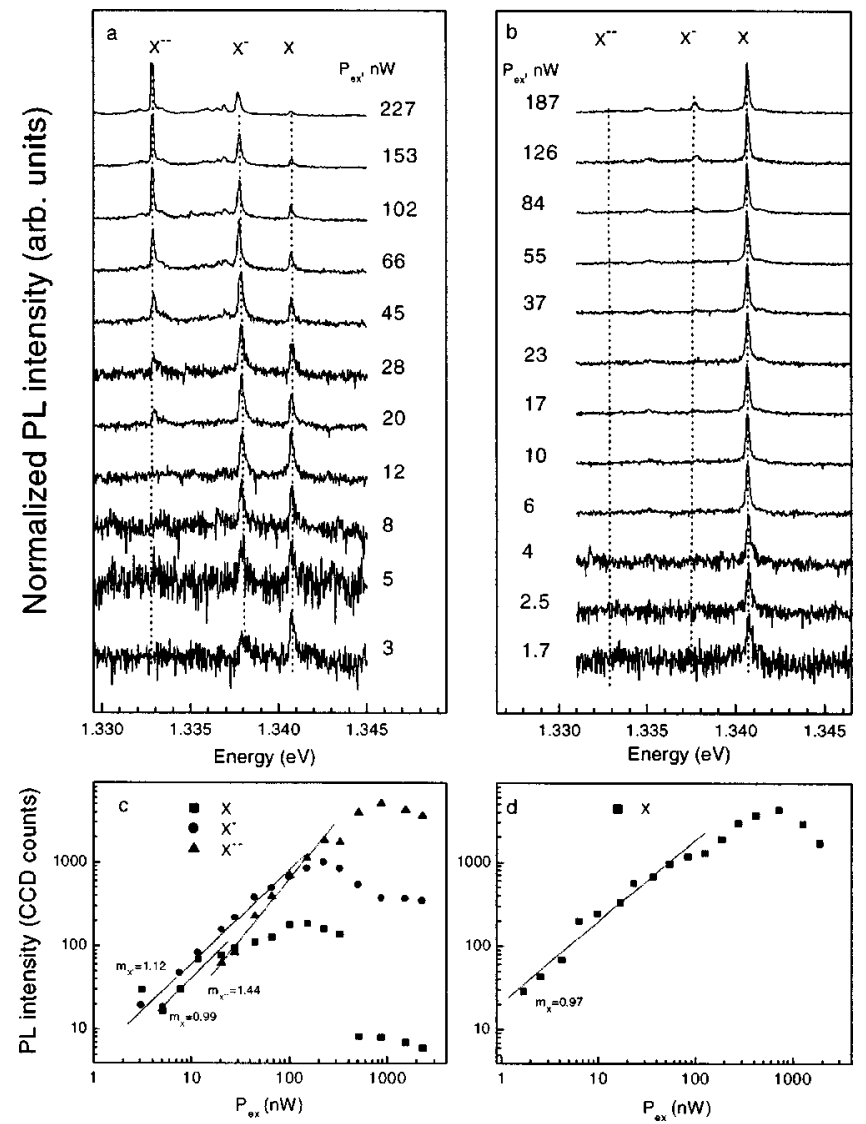

FIG. 4. PL spectra of a single QD1 taken at $T=4.1 \mathrm{~K}$, for a number of excitation powers (shown in the figure) and different excitation energies: (a) $h \nu_{\mathrm{ex}}=1.486 \mathrm{eV}$, (b) $h \nu_{\mathrm{ex}}=1.472 \mathrm{eV}$. All spectra are normalized at the maximum value of their PL amplitude and are vertically shifted for clarity. The integrated PL intensities of the detected PL lines, calculated for (c) $h \nu_{\mathrm{ex}}=1.486 \mathrm{eV}$ and (d) $h \nu_{\mathrm{ex}}=1.472 \mathrm{eV}, m_{i}\left(i=X, X^{-}, X^{--}\right)$give the slope of the corresponding fitting lines.

cluded. (ii) $h \nu_{\text {th }}$ energies have the same ( $P_{\text {ex }}$ dependent) values for different QD's within each group. At the same time, the ground (excited) state transition energies vary (by up to $40 \mathrm{meV}$ ) between different QD's within each group, thus totally excluding the resonant excitation conditions as prevailing in Ref. 43. (iii) The well-defined $h \nu_{\text {th }}$ energies differ between the two groups by $\approx 10 \mathrm{meV}$, while it was possible to find QD's belonging to the different groups with almost the same ground state transition energies (shifted by $\approx 1$ meV). (iv) $h \nu_{\text {ex }}$ reveals a $P_{\text {ex }}$ dependence (to be further exploited below), analogous for both groups of QD's [Fig. 3(a)], which could hardly be expected for the resonant phonon-assisted relaxation.

To get further insight on the origin of $h \nu_{\text {th }}$, we studied the $P_{\text {ex }}$ dependence of the PL spectra taken at a fixed $h \nu_{\text {ex }}$. This is shown in Fig. 4 for $T=4.1 \mathrm{~K}$ and (a) $h \nu_{\mathrm{ex}}=1.486 \mathrm{eV}$ and (b) $h \nu_{\mathrm{ex}}=1.472 \mathrm{eV}$, respectively. The PL spectra in Fig. 4(a) clearly reveal an intensity redistribution in favor of the $X^{-}$ and $X^{--}$lines. For $P_{\text {ex }}>200 \mathrm{nW}$, the line $X^{--}$dominates the spectra. This means that the QD becomes progressively more charged with increasing $P_{\mathrm{ex}}$. In sharp contrast, for 
spectra taken at $h \nu_{\text {ex }}<h \nu_{\text {th }}$ in the same $P_{\text {ex }}$ range [Fig. 4(b)], only the single $X$ line appears. Its intensity develops linearly with $P_{\mathrm{ex}}$ up to $P_{\mathrm{ex}} \approx 100 \mathrm{nW}$, at which saturation is initiated, to reach a maximum at $P_{\mathrm{ex}}=700 \mathrm{nW}$ and then slightly decreases with further increase of $P_{\text {ex }}$ [Fig. 4(d)].

This behavior can be explained in terms of an averaged exciton occupation number $N_{x}$ for a given QD. ${ }^{35} \mathrm{At} \mathrm{low} P_{\mathrm{ex}}$, $N_{x}<1$ and the amplitude of the $X$ line, which directly reflects the probability $W_{x}$ for the occupation of the QD with a configuration, corresponding to the neutral exciton $(1 e 1 h)$, develops linearly with $P_{\mathrm{ex}}$. With increasing excitation power, $N_{x}$ approaches $1, W_{x}$ reaches its maximum value, and consequently the $X$ line reveals its maximum amplitude. At even higher excitation power, when $N_{x}$ starts to exceed 1 , the probability to find a QD populated with the $2 e 2 h$ configuration increases at the expense of the neutral exciton $(1 e 1 h)$, which eventually results in a decreasing $X$ line amplitude ( $W_{x}$ progressively quenches). This is expected, since, at any given time, only one well-defined multiple exciton (configuration) may exist in the QD. For example, if the QD is occupied with two excitons ( $2 e 2 h$ configuration), the probability to find the QD with a single exciton $1 e 1 h$ and consequently the PL intensity of the line $X$ is expected to be zero. On the contrary, when a time-averaged (tens of seconds) spectrum, as used in the present study is recorded, several PL lines are detected. Each of them corresponds to a well-defined QD configuration, which exists at an instant given time. Thus, the experimentally derived dependence of the $X$ line on $P_{\text {ex }}$ [Fig. 4(d)] could be used as a reference to determine the evolution of $W_{x}$ with excitation power.

The $P_{\mathrm{ex}}$ dependence of the intensity of the $X$ line, taken at another $h \nu_{\mathrm{ex}}=1.486 \mathrm{eV}$, shown in Fig. 4(c), is in sharp contrast to the case illustrated in Fig. 4(d). Indeed, it exhibits a linear increase in a very restricted power range (up to 20 $\mathrm{nW}$ ), increases at a slower rate for higher $P_{\mathrm{ex}}$ to reach its maximum at $P_{\mathrm{ex}}=100 \mathrm{nW}$. The remarkable feature is that the $X$ line completely vanishes at $P_{\mathrm{ex}}>300 \mathrm{nW}$, i.e., at an excitation power, even below the maximum value of the $X$ line (and hence maximum value of $W_{x}$ ) in case of $h \nu_{\mathrm{ex}}$ $\left\langle h \nu_{\text {th }}\right.$ [Fig. 4(d)]. Accordingly, this observed rapid quenching of the PL amplitude of the line $X$ [Fig. 4(c)] is not explained by the increased probability of the QD occupation with $2 e 2 h$ with $P_{\mathrm{ex}}$, but rather the competing charged exciton, which gains intensity. The obvious difference between these two excitation energies is the extra electrons accumulated in the QD in the first case [Fig. 4(a)]. This anomalous behavior of the $X$ line can be readily understood if we, in addition to $W_{x}$, introduce probabilities for a QD to become charged with one $\left(W_{1}\right)$ or two $\left(W_{2}\right)$ extra electrons. Here we will just consider the case when the QD could be charged with up to two extra electrons, due to the fact that the PL spectra [Fig. 4(a)] reveal only two extra lines corresponding to charged excitons $\left(X^{-}\right.$and $\left.X^{--}\right)$in addition to the $X$ line. These charging probabilities obey the equation $W_{0}+W_{1}$ $+W_{2}=1$, where $W_{0}$ corresponds to the probability to find the QD empty. This equation follows from the simple consideration that an exciton, captured into the QD, can be faced with only three different situations: The QD is empty or filled with 1 or 2 electrons. Correspondingly, the PL intensities $I_{X}, I_{X^{-}}$, and $I_{X^{--}}$of the spectral lines $X, X^{-}$, and $X^{--}$ are given by $W_{x} \times W_{0}, W_{x} \times W_{1}$, and $W_{x} \times W_{2}$, respectively. The above revealed redistribution of the PL spectra [Fig. 4(a)] means that with increasing $P_{\text {ex }}$, the values of $W_{1}$ and $W_{2}$ progressively increase at the expense of $W_{0}$ and at $P_{\text {ex }}$ $>300 \mathrm{nW}$ the latter becomes negligible. This fact explains the disappearance of the $X$ line in PL at $P_{\mathrm{ex}}>300 \mathrm{nW}$ nicely, despite of a progressive increase of $W_{x}$ in the power range up to $700 \mathrm{nW}$. It should be mentioned, that deriving the $W_{x}$ from the power evolution of the $X$ line [Fig. 4(d)], we implicitly imposed the condition $W_{0}=1, W_{1}=W_{2}=0$, which is justified by the absence of the lines $X^{-}$and $X^{--}$in the PL spectra in Fig. 4(b).

The $X^{-}$line increases in intensity up to $P_{\mathrm{ex}}=200 \mathrm{nW}$, but decreases at higher powers. The $X^{--}$line exhibits an analogous behavior, except for an increasing intensity up to a considerably higher excitation intensity $P_{\mathrm{ex}} \approx 750 \mathrm{nW}$, which approximately coincides with the $P_{\text {ex }}$ value, corresponding to the maximum $W_{x}$, derived from the data shown in Fig. 4(d). In terms of the above introduced charging probabilities, this behavior means that for $P_{\mathrm{ex}}>200 \mathrm{nW}$, the probability to find a QD loaded with more than one extra electron is essential and increases progressively at the expense of $W_{1}$ and $W_{0}$. It is interesting to note that the integrated intensity of the $X^{-}$ line $I_{X^{-}}$shows a slightly superlinear increase $\left(I_{X^{-}} \sim P_{\mathrm{ex}}^{1.12}\right)$ at $P_{\text {ex }} \leqslant 150 \mathrm{nW}$ similarly to the $X^{--}$line characterized by $I_{X^{--}} \sim P_{\mathrm{ex}}^{1.44}$ at $P_{\mathrm{ex}} \leqslant 200 \mathrm{nW}$. Accordingly, in order to observe the $X^{-}$and $X^{--}$lines, absorption of more than one photon on average is needed.

In contrast to these findings, an exactly linear power dependence of the charged exciton line was reported in Refs. 36 and 38. In our previous studies of the same QD, ${ }^{31}$ performed at other excitation conditions $\left(h \nu_{\mathrm{ex}}>E_{g}^{\mathrm{GaAs}}\right)$, we also found a simultaneous linear increase, saturation and decrease for all the $I_{X}, I_{X^{-}}$, and $I_{X^{--}}$with increasing $P_{\mathrm{ex}}$. The charging mechanism proposed in Ref. 31 was based on the different diffusivities of $e$ 's and $h$ 's, which could be achieved at certain $h \nu_{\mathrm{ex}}$ values, and hence an essentially faster capture processes for the electrons (compared to the holes) into the QD. Consequently we implicitly assumed a capture process limited by the diffusion mechanism, i.e., an exact value of the $h \nu_{\text {ex }}$ determined the ratio $W_{0}: W_{1}: W_{2}$, which was not dependent on $P_{\mathrm{ex}}$. This mechanism could explain the existence of the small contribution of the $X^{-}$ line, which progressively decreases in amplitude as $h \nu_{\mathrm{ex}}$ decreases from $h \nu_{\text {th }}$ down to $E_{\mathrm{WL}}$ [Fig. 1(a)]. Our present findings evidently imply a completely different charging mechanism, which should take into account both the existence of a threshold energy and a high sensitivity to changes in $P_{\mathrm{ex}}$.

To explain the experimental results obtained we propose the following model. The laser excitation with $h \nu_{\mathrm{ex}}>h \nu_{\text {th }}$ will create equal number of $e$ 's and $h$ 's, absorbed in the WL (arrow 2 and 3 in Fig. 2), but will in addition photogenerate some free electrons in the surrounding barriers as a result of the light absorption by the ionized acceptor atoms, which are present in the barrier as residual dopants (arrow 4 in Fig. 2). Then, the $h \nu_{\text {th }}$ has to be equal to the $E_{g}^{\mathrm{GaAs}}-E_{a}$, where $E_{a}$ is 
the acceptor binding energy. In fact the $h \nu_{\text {th }}$ values, obtained for high $P_{\text {ex }}$ for the two groups of QD's, would imply $E_{a}$ to be equal to 36 (26) meV for the first (second) QD's group, respectively. These values are similar to the $\mathrm{Si}(\mathrm{C})$ acceptor binding energies of $34(25.5) \mathrm{meV}^{44}$

The extra electrons created in the GaAs barriers, are expected to be effectively captured into the WL (and subsequently into the QD) due to their very efficient transport in GaAs crystals ${ }^{39}$ accompanied by the absence of free holes in the barriers at $h \nu_{\mathrm{ex}}<E_{g}^{\mathrm{GaAs}}$. This model, indeed, implies that to populate the QD with a charged exciton configuration, absorption of at least two photons is needed. One of them excites an exciton inside the WL (processes, illustrated by arrows 2 and 3 in Fig. 2) and the second should excite an electron in the GaAs barrier (arrow 4 in Fig. 2). This results in an expected superlinear dependence $\left(\sim P_{\mathrm{ex}}^{m}\right)$ of the PL amplitude of the charged exciton lines on $P_{\mathrm{ex}}$. When an extra electron, loaded into the QD, remains there for a time, $\tau_{e}$, much longer than the time interval $\tau_{x}$ between two sequential events of the $e-h$ pair (excited in the WL) capture into the QD, then $m$ should be close to 1 . In case of a shorter $\tau_{e}, m$ will exceed 1 with an expected maximum, $m=2$, when two photons are required to generate each charged exciton. In our case, we are in the intermediate range between these two extreme cases, since the derived values are $m_{X^{-}}$ $=1.12$ and $m_{X^{--}}=1.44$ [Fig. 4(c)].

Increasing $P_{\mathrm{ex}}$ results in the progressive loading of the QD with extra electrons, redistributing the $W_{i}$ 's $(i=0,1,2)$ in favor of $W_{2}$. The $W_{i}$ 's were obtained directly from the experimental results [Fig. 4(c)] according to $W_{0}=I_{X} /\left(I_{X}\right.$ $\left.+I_{X^{-}}+I_{X^{--}}\right), \quad W_{1}=\left(I_{X^{-}}\right) /\left(I_{X}+I_{X^{-}}+I_{X^{--}}\right), \quad$ and $W_{2}$ $=\left(I_{X^{--}}\right) /\left(I_{X}+I_{X^{-}}+I_{X^{--}}\right)$. The results are shown in Fig. 5(a) as a function of $P_{\mathrm{ex}}$. Taking into account that $W_{1}$ $\left(W_{2}\right)$ represents the probability to find the QD loaded with one (two) extra electron(s), the calculation of the total charge accumulated in the QD is rather straightforward and given by $W_{1}+2 \times W_{2}$ [Fig. 5(b)]. According to the model presented, $W_{1}+2 \times W_{2}$ should be proportional to the total number of extra electrons $n^{b}$, photoexcited in the barrier and, as a consequence, must directly reflect the power evolution of $n^{b}$.

The calculated absorption coefficient for the acceptor-toconduction band transition for bulk $\mathrm{GaAs}^{45}$ was found to be proportional to $\left(E_{c}\right)^{1 / 2}$, where $E_{c}=h \nu_{\mathrm{ex}}-\left(E_{g}^{\mathrm{GaAs}}-E_{a}\right)$ is the kinetic energy of the photocreated electron in the GaAs, simply reflecting the 3D density of states of the conduction band. (We note here that the GaAs barriers in the sample studied are 100-nm thick and hence can be regarded as bulklike.) Consequently, when $h \nu_{\mathrm{ex}}$ decreases to $E_{g}^{\mathrm{GaAs}}-E_{a}$, the absorption approaches zero. Hence, a high $P_{\mathrm{ex}}$ is needed to create $n^{b}$, sufficient to result in threshold phenomenon. In contrary, when $h \nu_{\text {th }}\left(E_{c}\right)$ progressively increases, a relatively smaller value of $P_{\mathrm{ex}}$ would be enough to create the same number of $n^{b}$ and, correspondingly, load the QD with the same number of extra charge as in case of small $E_{c}$. These circumstances explain the redshift of the $h \nu_{\text {th }}$ with $P_{\text {ex }}$, detected in the experiment for the both groups of QD's [Fig. $3(\mathrm{a})]$.

To calculate $n^{b}=n \times V_{b}$, where $n$ is the photoexcited
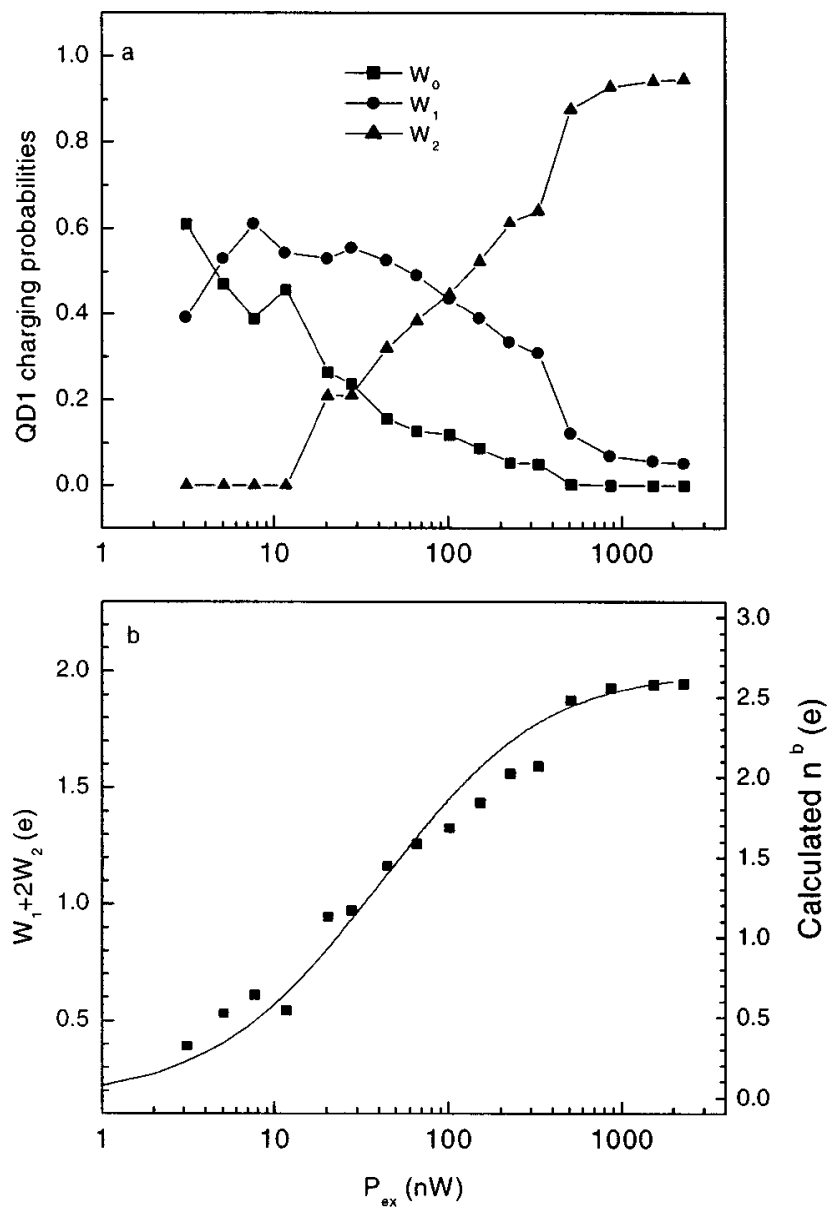

FIG. 5. The QD1 charging probabilities $W_{0}, W_{1}$, and $W_{2}$ (a) and a total charge accumulated in the QD $W_{1}+2 W_{2}$ [(b) left vertical axis] as a function of $P_{\text {ex }}$, taken at $T=4.1 \mathrm{~K}$ and $h \nu_{\text {ex }}$ $=1.486 \mathrm{eV}$. The solid line in (b) represents the results of a calculated value of $n^{b}$ (right axis).

steady-state electron concentration in the GaAs and $V_{b}=8$ $\times 10^{-13} \mathrm{~cm}^{3}$ is the volume of the barrier excited by the laser light, we apply a simple rate equation, which accounts for the electron generation from the ionized acceptors (with concentration $N_{a}^{-}$) and the reverse recombination process to neutral acceptors $\left(N_{a}^{0}\right)$ :

$$
d n / d t=+g_{0} \times \alpha_{0} \times N_{a}^{-}-\left(n \times N_{a}^{0}\right) / \tau_{0},
$$

where $g_{0}$ is the number of incident photons per area and time unit, $\alpha_{0}=1.2 \times 10^{-15} \mathrm{~cm}^{2}$, which is calculated for $h \nu_{\mathrm{ex}}$ $=1.486 \mathrm{eV}$ and $E_{c}=3 \mathrm{meV},{ }^{45} \tau_{0}=2 \times 10^{9} \mathrm{~s} \mathrm{~cm}^{-3}$, calculated for $T=4.1 \mathrm{~K}^{45}$ The barrier material is $p$ type $^{46}$ with a compensation degree $k=N_{d} / N_{a}<1$, where $N_{d}\left(N_{a}\right)$ is the total concentration of donor (acceptor) atoms. Consequently, we consider the total number of ionized acceptors at $g_{0}=0$ and $T=4.1 \mathrm{~K}$ to be equal to $k \times N_{a}$. To solve the Eq. (1), a distribution function $f$ has been introduced, which accounts for the probability for $k \times N_{a}$ atoms to be filled with electrons at $g_{0} \neq 0$. Then, the neutrality condition can be expressed in the form 


$$
n=k \times N_{a} \times(1-f)
$$

and, correspondingly,

$$
\begin{gathered}
N_{a}^{-}=f \times k \times N_{a}, \\
N_{a}^{0}=k \times N_{a} \times(1-f)+N_{a} \times(1-k) .
\end{gathered}
$$

Taking into account Eqs. (2) and (3), the steady-state solution of Eq. (1) has been obtained and is shown as a function of $P_{\text {ex }}$ in Fig. 5(b). The calculations are based on an acceptor concentration of $N_{a}=10^{13} \mathrm{~cm}^{-3}$ and $k=\frac{1}{3}$ as adjustable parameters. It is clearly seen, that the calculated curve rather nicely reproduces the $W_{1}+2 \times W_{2} P_{\text {ex }}$ dependence. (The maximum value of $N_{a}^{-}$used is 3.3 $\times 10^{12} \mathrm{~cm}^{-3}$, which approximately corresponds to the three ionized acceptor atoms in the excited volume of the crystal.) It should be pointed out that even at such a low acceptor concentration it is still possible to create $n^{b}$, which is sufficient to load the QD under study with up to two extra electrons.

This fact is valid under the assumption that the QD can collect most of the $n^{b}$ electrons (in the upper limit-all), generated in the GaAs within the laser spot, which is $\approx 2 \mu \mathrm{m}$ in diameter. To check this idea, we performed space-resolved experiments, in which PL spectra, taken with the laser spot positioned on the QD, were compared with spectra detected with the laser spot moved aside of the dot. The spectra are shown in Fig. 6 a for the case of excitation with a $h \nu_{\text {ex }}$ $>E_{g}^{\mathrm{GaAs}}$, generated in such a way that an equal number of $e$ 's and $h$ 's are created in the sample. The above discussed acceptor model has no effect here due to the negligible value of $N_{a}$ with respect to the values of the 3D density of states involved in the band-to-band transitions. $h \nu_{\mathrm{ex}}$ was chosen ${ }^{31}$ in such a way that the PL spectrum involves both the neutral and charged exciton lines [the upper part of Fig. 6(a)]. It is clearly seen, that only the $X$ line is observed in the PL, when the laser spot was moved aside of the QD by $2.5 \mu \mathrm{m}$.

These dramatic changes can be explained as follows. As already discussed above, the QD becomes negatively charged although the total number of photoexcited electrons and holes are equal, due to the faster capture of $e$ 's into the QD. These processes are effective when the QD is positioned in the center of the laser spot: The electrons move faster than the holes and are accordingly faster captured into the QD. Still the total charge neutrality is not broken within the laser spot. On the contrary, when the laser spot is positioned next to the QD, the propagation of photoexcited electrons and holes from the excitation spot in pairs (as excitons) is expected, because the hole mobilities will limit the transport of photocreated $e-h$ pairs in GaAs. ${ }^{47}$ As a result, the QD is expected to be populated with only neutral excitons as is clearly demonstrated in the lower part of Fig. 6(a).

In striking contrast to these observations, at excitation with $h \nu_{\mathrm{th}}<h \nu_{\mathrm{ex}}<E_{g}^{\mathrm{GaAs}}$, the PL spectra remain the same for both excitation conditions [Fig. 6(b)]. According to the above conclusions this could be realized only for the case when the excitation produces nonequal amount of free electrons and holes. Indeed, if in addition to the equal number of

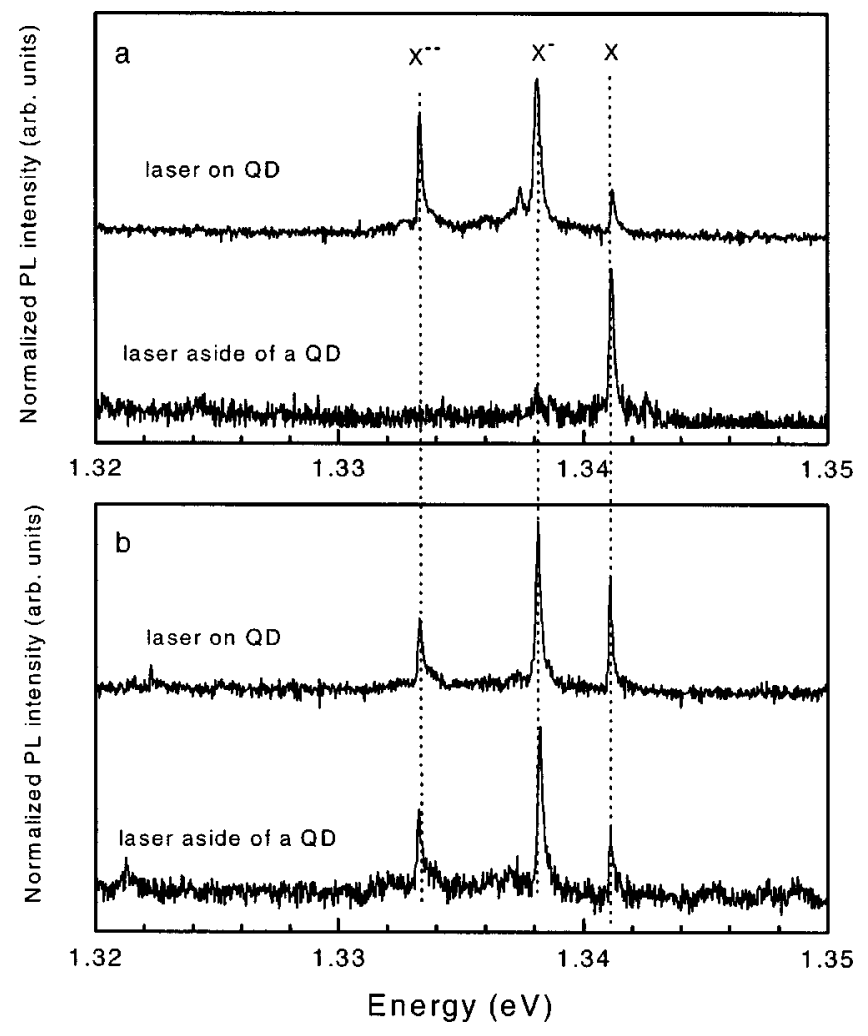

FIG. 6. PL spectra of a single QD1 taken at $T=4.1 \mathrm{~K}$ and (a) $h \nu_{\mathrm{ex}}=1.560 \mathrm{eV}, \quad P_{\mathrm{ex}}=60 \mathrm{nW}$ and (b) $h \nu_{\mathrm{ex}}=1.486 \mathrm{eV}, \quad P_{\mathrm{ex}}$ $=50 \mathrm{nW}$ for the different excitation geometries: The laser spot is positioned on a $[2.5 \mu \mathrm{m}$ side of (a)] QD shown in the upper (lower) parts in (a) and (b), respectively. All spectra are normalized at the maximum value of their PL amplitude and are vertically shifted for clarity. The integrated PL intensities between each pair of spectra differ by a factor of 9.2 (a) and 3.2 (b).

$e^{\prime} \mathrm{s}$ and $h$ 's photoexcited in the WL, some extra electrons $n^{b}$ are excited in the GaAs barriers, these charge carriers will eventually reach the QD to generate a negatively charged QD. The same amplitude ratio between the three lines in Fig. 6(b) for both "geometries" clearly indicates the loading of the QD with the same number of extra electrons, which proves the QD's ability to collect extra charges from a distance, exceeding the size of the laser spot.

To further elucidate the role of the experimental conditions on the acceptor-induced charging mechanism of a QD, PL spectra were detected for a range of sample temperatures for excitations with $h \nu_{\mathrm{th}}<h \nu_{\mathrm{ex}}<E_{g}^{\mathrm{GaAs}}$ and $E_{\mathrm{WL}}<h \nu_{\mathrm{ex}}$ $<h \nu_{\text {th }}$ [Figs. 7(a) and 7(b)], respectively. The evident gradual disappearance of the $X^{--}$line with increasing $T$ is clearly revealed for $h \nu_{\text {th }}<h \nu_{\text {ex }}<E_{g}^{\mathrm{GaAs}}$ [Fig. 7(a)]. At highest temperatures employed ( $T=49 \mathrm{~K})$, the PL spectrum consists only of the $X^{-}$line. In sharp contrast, for $E_{\mathrm{WL}}<h \nu_{\mathrm{ex}}$ $\left\langle h \nu_{\text {th }}\right.$ the opposite behavior is observed [Fig. 7(b)], for which the increase of $T$ leads to the redistribution of the PL intensities in favor of the $X^{-}$line, while a monotonous redshift of the entire spectra is observed for both cases [Figs. 7 (a) and 7(b)]. At high $T$, the spectra for the two excitation energies are the same [Figs. 7(a) and 7(b)]. This means, that 


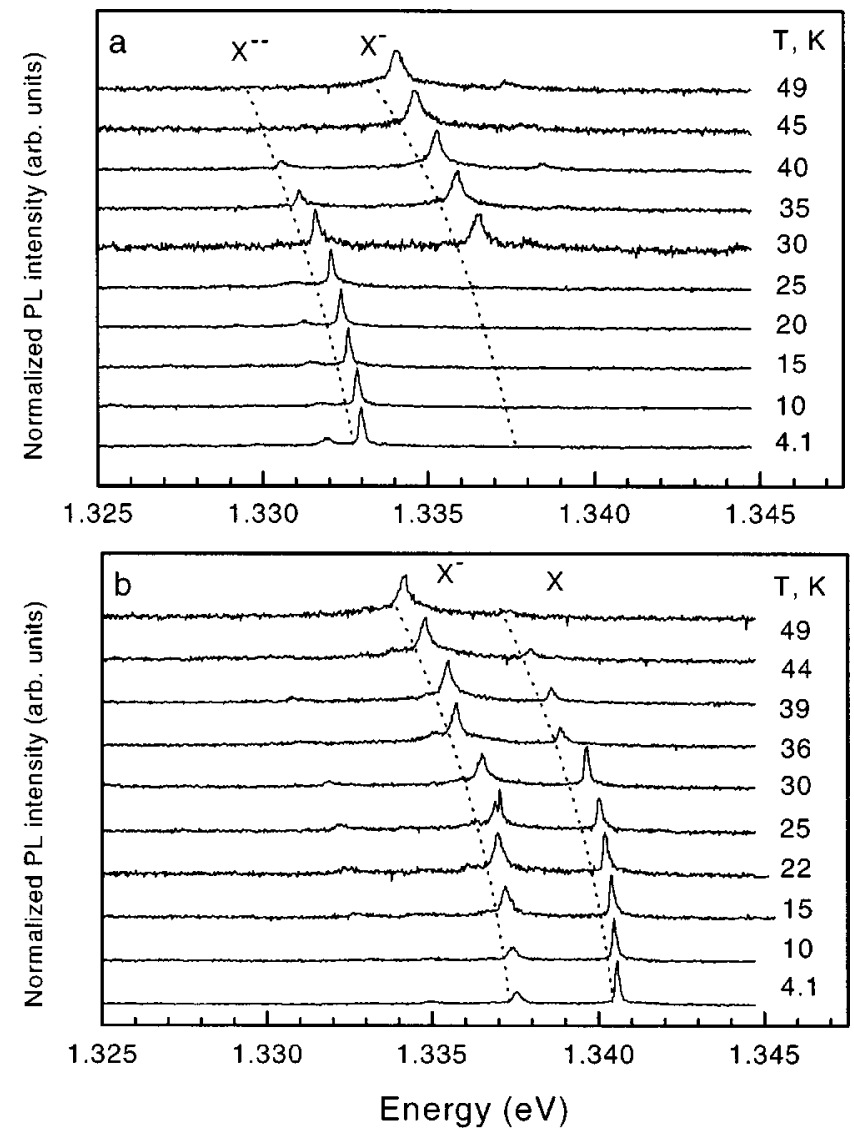

FIG. 7. PL spectra of a single QD1 taken at a number of temperatures (shown in the figure) and different excitation conditions (a) $h \nu_{\mathrm{ex}}=1.501 \mathrm{eV}, P_{\mathrm{ex}}=50 \mathrm{nW}$ and (b) $h \nu_{\mathrm{ex}}=1.471 \mathrm{eV}, P_{\mathrm{ex}}$ $=80 \mathrm{nW}$. All spectra are normalized at the maximum value of their PL amplitude and are vertically shifted for clarity. The dotted lines are guides for the eye.

the threshold phenomenon is not observed at elevated temperatures. Indeed, the measurements, analogous to those shown in Fig. 1(a), but performed at $T=50 \mathrm{~K}$ (not shown here) clearly demonstrate the absence of the threshold.

The evolution of PL spectra with increasing $T$ taken at $h \nu_{\mathrm{ex}}>E_{g}^{\mathrm{GaAs}}$ has been studied previously. ${ }^{32}$ It was demonstrated that at any $h \nu_{\mathrm{ex}}>E_{g}^{\mathrm{GaAs}}$, the peak intensities in the spectra are redistributed in favor of $X^{-}$and $X^{--}$lines at increased $T$. Already at $T=T_{0}=35-40 \mathrm{~K}$, the spectra are dominated by the $X^{-}$and $X^{--}$lines. This behavior is explained in terms of a temperature-induced increased electron diffusivity in the GaAs barriers, which eventually leads to a faster electron capture into the QD. This behavior is consistent with our present results [shown in Fig. 7(b)], except one thing. Even at $T>T_{0}$, the $X^{--}$line cannot be detected. The evolution of the PL spectra with increasing $T$ [Fig. 7(a)] is totally different, inevitably indicating that some other temperature-induced charging/discharging mechanism has to be considered.

The above discussed model of an acceptor-induced charging predicts the observed dependence on the temperature nicely. Indeed, the increase of the temperature enhances the role of the acceptor ionization processes from the valence band of the GaAs. On one hand, it should lead to an increase of $n^{b}$ due to the increased total number of $N_{a}^{-}$. On the other hand, the presence of free holes in the valence band of GaAs, will result in an extremely efficient (compared to the capture of photocreated $n^{b}$ back to the acceptors) band-to-band recombination mechanism of $n^{b}$. Indeed, the typical radiative recombination times for the free-to-free transitions are of the order of $1 \mathrm{~ns},{ }^{45}$ while that for the free-to-acceptor transitions in GaAs are of the order of $100 \mu$ s calculated for $N_{a}$ $=10^{13} \mathrm{~cm}^{-3} .45$ Thus, the appearance of the free holes with increasing temperature is expected to effectively decrease the $n^{b}$, which in turn should result in a reduced charging (total discharging) of the QD. This prediction of the acceptor model is in full agreement with our experimental observations [Fig. 7(a)]. At the same time, the temperature-generated extra concentration of free holes $p$ in GaAs could explain the $X^{--}$line absence at $T>T_{0}$ observed in Fig. 7(b).

To estimate the characteristic temperature $T^{*}$, above which $n^{b}$ should be considerably decreased with respect to the values, calculated for $T=4.1 \mathrm{~K}$, an additional term $-\gamma$ $\times n \times p$, which accounts for the band-to-band recombination rate, has to be introduced in the right-hand part of Eq. (1), where $\gamma=\gamma_{0} \times T^{-2}$ is the recombination coefficient ${ }^{48}$ and $\gamma_{0} \approx 9 \times 10^{-5} \mathrm{~cm}^{3} \mathrm{~s}^{-1} \mathrm{~K}^{2}$ could be calculated for bulk GaAs. ${ }^{48}$ Then, $n$ can be expressed as

$$
n=g_{0} \times \alpha_{0} \times \tau_{0} \times N_{a}^{-} /\left(N_{a}^{0}+\gamma \times \tau_{0} \times p\right) .
$$

The quantity of $p$ could be roughly calculated using the expression $p=\left(N_{v} \times N_{a}^{0} / 2\right)^{1 / 2} \times \exp \left(-E_{a} / 2 k_{B} T\right),{ }^{48}$ where $N_{v}$ $=2\left(2 \pi m_{h}^{*}\right)^{3 / 2} \times\left(k_{B} T\right)^{3 / 2} / h^{3}$ is the effective density of states of the valence band for bulk material and $m_{h}^{*}=0.45 m_{0}\left(m_{0}\right.$ is the free electron mass) is the hole effective mass in GaAs. ${ }^{39}$ At $\beta=\gamma \times \tau_{0} \times p / N_{a}^{0} \gg 1$, according to Eq. (4), bandto-band transitions will be the dominant mechanism, which determines the steady-state value of $n$. We thus derived $T^{*}$ $=24 \mathrm{~K}$ provided $\beta=10$. It is readily seen [Fig. $7(\mathrm{a})]$ that the PL spectra start to become more "neutral" at $T>25 \mathrm{~K}$, which agrees well with the estimated $T^{*}$ value. Our calculations also result in a decrease of $n\left(n^{b}\right)$ by one order of magnitude at $T=40-50 \mathrm{~K}$ compared with the calculated $n^{b}$ values at $T=4.1 \mathrm{~K}$ [Fig. 5(b)].

\section{CONCLUSION}

The threshold phenomenon regarding the excitation conditions for the QD's charging has been revealed from PL spectra of single InAs/GaAs self-assembled quantum dots. A well-defined excitation energy, below (above) which the QD is in the neutral (charged) state is manifested. The studies of this phenomenon for a number of different QD's and at different experimental conditions (such as $h \nu_{\mathrm{ex}}, P_{\mathrm{ex}}$, and $T$ ) allow us to make a definite conclusion about the particular mechanism responsible for the loading of the QD with up to two extra electrons. This is considered to be the acceptorrelated transitions resulting in a photoexcitation of negative charge in the barrier material, which was directly proved by the spatially resolved experimental findings. The latter fact 
together with the results of the calculations show that the QD can really be loaded with two extra electrons even at a very low $\left(\sim 10^{13} \mathrm{~cm}^{-3}\right)$ concentration of residual dopants. The acceptor-induced charging phenomenon is suggested to be used in practice as an effective tool to create and study charged versus neutral excitons in QD's.

\section{ACKNOWLEDGMENTS}

One of us (E.S.M.) gratefully acknowledges financial support from the Wenner-Gren Foundations and partial support from the Russian Academy of Sciences (Low-Dimensional Nanostructures' 2001).
${ }^{1}$ D. Gammon, Nature (London) 405, 899 (2000).

${ }^{2}$ G. Medeiros-Ribeiro, F. G. Pikus, P. M. Petroff, and A. L. Efros, Phys. Rev. B 55, 1568 (1997).

${ }^{3}$ A. Wojs and P. Hawrylak, Phys. Rev. B 55, 13066 (1997).

${ }^{4} \mathrm{Ph}$. Lelong and G. Bastard, Solid State Commun. 98, 819 (1996).

${ }^{5}$ R. J. Warburton, B. T. Miller, C. S. Durr, C. Bodefeld, K. Karrai, J. P. Kotthaus, G. Medeiros-Ribeiro, P. M. Petroff, and S. Huant, Phys. Rev. B 58, 16221 (1998).

${ }^{6}$ A. Wojs, P. Hawrylak, S. Faffard, and L. Jacak, Phys. Rev. B 54, 5604 (1996).

${ }^{7}$ G. A. Narvaez and P. Hawrylak, Phys. Rev. B 61, 13753 (2000).

${ }^{8}$ A. Orlandi, M. Rontani, G. Goldoni, F. Manghi, and E. Molinari, Phys. Rev. B 63, 045310 (2001).

${ }^{9}$ A. Franceschetti and A. Zunger, Phys. Rev. B 62, 2614 (2000).

${ }^{10}$ M. Bayer, T. Gutbrod, A. Forchel, V. D. Kulakovskii, A. Gorbunov, M. Michel, R. Steffen, and K. H. Wang, Phys. Rev. B 58, 4740 (1998).

${ }^{11}$ E. Dekel, D. Gershoni, E. Ehrenfreund, D. Spector, J. M. Garcia, and P. M. Petroff, Phys. Rev. Lett. 80, 4991 (1998).

${ }^{12}$ K. Brunner, G. Abstreiter, G. Bohm, G. Trankle, and G. Weinmann, Phys. Rev. Lett. 73, 1138 (1994).

${ }^{13}$ L. Landin, M. E. Pistol, C. Pryor, L. Samuelson, and M. Miller, Phys. Rev. B 60, 16640 (1999).

${ }^{14}$ S. Tarucha, D. G. Austing, T. Honda, R. J. van der Hage, and L. P. Kouwenhoven, Phys. Rev. Lett. 77, 3613 (1996).

${ }^{15}$ K. H. Schmidt, G. Medeiros-Ribeiro, M. Oestreich, P. M. Petroff, and G. H. Dohler, Phys. Rev. B 54, 11346 (1996); K. H. Schmidt, G. Medeiros-Ribeiro, and P. M. Petroff, ibid. 58, 3597 (1998).

${ }^{16}$ H. Drexler, D. Leonard, W. Hansen, J. P. Kotthaus, and P. M. Petroff, Phys. Rev. Lett. 73, 2252 (1994).

${ }^{17}$ R. J. Warburton, C. S. Durr, K. Karrai, J. P. Kotthaus, G. Medeiros-Ribeiro, and P. M. Petroff, Phys. Rev. Lett. 79, 5282 (1997).

${ }^{18}$ B. T. Miller, W. Hansen, S. Manus, R. J. Luyken, A. Lorke, J. P. Kotthaus, and S. Huant, Phys. Rev. B 56, 6764 (1997).

${ }^{19}$ W. H. Chang, W. Y. Chen, M. C. Cheng, C. Y. Lai, T. M. Hsu, N. T. Yeh, and J. I. Chyi, Phys. Rev. B 64, 125315 (2001).

${ }^{20}$ K. Goede, A. Weber, F. Guffarth, C. M. A. Kapteyn, F. Heinrichsdorff, R. Heitz, D. Bimberg, and M. Grundmann, Phys. Rev. B 64, 245317 (2001).

${ }^{21}$ P. Paskov, P. O. Holtz, S. Wongmanerod, B. Monemar, J. M. Garcia, W. V. Schoenfeld, and P. M. Petroff, Physica E 6, 440 (2000).

${ }^{22}$ L. Harris, D. J. Mawbray, M. S. Skolnick, M. Hopkinson, and G. Hill, Appl. Phys. Lett. 73, 969 (1998).

${ }^{23}$ S. Maimon, E. Finkman, G. Bahir, S. E. Schacham, J. M. Garsia, and P. M. Petroff, Appl. Phys. Lett. 73, 2003 (1998).

${ }^{24}$ J. J. Finley, M. Skalitz, M. Arzberger, A. Zrenner, G. Bohm, and
G. Abstreiter, Appl. Phys. Lett. 73, 2618 (1998).

${ }^{25}$ T. Lundstrom, W. Schoenfeld, H. Lee, and P. M. Petroff, Science 286, 2312 (1999).

${ }^{26}$ D. Bimberg, M. Grundmann and N. N. Ledentsov, Quantum Dot Heterostructures (Wiley, London, 1999).

${ }^{27}$ M. Bayer, A. Kuther, F. Schafer, J. P. Reithmaier, and A. Forchel, Phys. Rev. B 60, R8481 (1999).

${ }^{28}$ A. Hartmann, Y. Ducommun, E. Kapon, U. Hohenester, and E. Molinari, Phys. Rev. Lett. 84, 5648 (2000).

${ }^{29}$ A. Hartmann, Y. Ducommun, M. Bachthold, and E. Kapon, Physica E 7, 461 (2000).

${ }^{30}$ R. J. Warburton, C. Schaflein, F. Haft, F. Bickel, A. Lorke, K. Karrai, J. M. Garsia, W. Schoenfeld, and P. M. Petroff, Nature (London) 405, 926 (2000).

${ }^{31}$ E. S. Moskalenko, K. F. Karlsson, P. O. Holtz, B. Monemar, W. V. Schoenfeld, J. M. Garsia, and P. M. Petroff, Phys. Rev. B 64, 085302 (2001).

${ }^{32}$ K. F. Karlsson, E. S. Moskalenko, P. O. Holtz, B. Monemar, W. V. Schoenfeld, J. M. Garsia, and P. M. Petroff, Appl. Phys. Lett. 78, 2952 (2001).

${ }^{33}$ M. Baier, F. Findeis, A. Zrenner, M. Bichler, and G. Abstreiter, Phys. Rev. B 64, 195326 (2001); F. Findeis, M. Baier, A. Zrenner, M. Bichler, G. Abstreiter, U. Hohenester, and E. Molinari, ibid. 63, 121309 (2001).

${ }^{34}$ D. Hessman, J. Persson, M. E. Pistol, C. Pryor, and L. Samuelson, Phys. Rev. B 64, 233308 (2001).

${ }^{35}$ D. V. Regelman, E. Dekel, D. Gershoni, E. Ehrenfreud, A. J. Williamson, J. Shumway, A. Zunger, W. V. Schoenfeld, and P. M. Petroff, Phys. Rev. B 64, 165301 (2001).

${ }^{36}$ R. M. Thompson, R. M. Stevenson, A. J. Shields, I. Farrer, C. J. Lobo, D. A. Ritchie, M. L. Leadbeater, and M. Pepper, Phys. Rev. B 64, 201302 (2001).

${ }^{37}$ A. Lemaitre, A. D. Ashmore, J. J. Finley, D. J. Mowbray, M. S. Skolnick, M. Hopkinson, and T. F. Krauss, Phys. Rev. B 63, 161309 (2001).

${ }^{38}$ J. J. Finley, A. D. Ashmore, A. Lemaitre, D. J. Mowbray, M. S. Skolnick, I. E. Itskevich, P. A. Maksym, M. Hopkinson, and T. F. Krauss, Phys. Rev. B 63, 073307 (2001).

${ }^{39}$ J. P. Wolfe, H. W. Yoon, D. R. Wake, and H. Morkoc, Semicond. Sci. Technol. 7, B240 (1992); H. W. Yoon, D. R. Wake, and J. P. Wolfe, Phys. Rev. B 54, 2763 (1996).

${ }^{40}$ P. C. Sersel, Phys. Rev. B 51, 14532 (1995).

${ }^{41}$ K. H. Schmidt, G. Medeiros-Ribeiro, J. M. Garcia, and P. M. Petroff, Appl. Phys. Lett. 70, 1727 (1997); J. M. Garcia, T. Mankad, P. O. Holtz, P. J. Wellman, and P. M. Petroff, ibid. 72, 3172 (1998); J. M. Garsia, G. Medeiros-Ribeiro, K. Schmidt, T. Ngo, J. L. Feng, A. Lorke, J. Kotthaus, and P. M. Petroff, ibid. 71, 2014 (1997).

${ }^{42}$ O. Brandt, H. Lage, and K. Ploog, Phys. Rev. B 45, 4217 (1992). 
${ }^{43}$ R. Heitz, M. Veit, N. N. Ledentsov, A. Hoffmann, D. Bimberg, V. M. Ustinov, P. S. Kop'ev, and Zh. I. Alferov, Phys. Rev. B 56, 10435 (1997).

${ }^{44}$ D. J. Ashen, P. J. Dean, D. T. J. Hurle, J. B. Mullin, A. M. White, and P. D. Greene, J. Phys. Chem. Solids 36, 1041 (1975).

${ }^{45}$ W. P. Dumke, Phys. Rev. 132, 1998 (1963).
${ }^{46}$ T. M. Kerr, C. E. C. Wood, S. M. Newstead, and J. D. Wilcox, J. Appl. Phys. 65, 2673 (1989).

${ }^{47}$ H. W. Yoon, D. R. Wake, J. P. Wolfe, and H. Morkoc, Phys. Rev. B 46, 13461 (1992).

${ }^{48}$ K. W. Böer, Survey of Semiconductor Physics (Van Nostrand Reinhold, New York, 1990). 